\title{
REVIEW ARTICLE OPEN The promise of mRNA vaccines: a biotech and industrial perspective
}

\author{
Nicholas A. C. Jackson ${ }^{1 *}$, Kent E. Kester $\mathbb{D}^{2}$, Danilo Casimiro ${ }^{2}$, Sanjay Gurunathan ${ }^{2}$ and Frank DeRosa ${ }^{3}$
}

mRNA technologies have the potential to transform areas of medicine, including the prophylaxis of infectious diseases. The advantages for vaccines range from the acceleration of immunogen discovery to rapid response and multiple disease target manufacturing. A greater understanding of quality attributes that dictate translation efficiency, as well as a comprehensive appreciation of the importance of mRNA delivery, are influencing a new era of investment in development activities. The application of translational sciences and growing early-phase clinical experience continue to inform candidate vaccine selection. Here we review the state of the art for the prevention of infectious diseases by using mRNA and pertinent topics to the biotechnology and pharmaceutical industries.

npj Vaccines (2020)5:11; https://doi.org/10.1038/s41541-020-0159-8

\section{INTRODUCTION}

Continued growth in the vaccine business is expected based on expanded coverage, improved existing products, and new vaccines. Among other factors, manufacturing must change to support growth. Capital-rich investment in fixed facilities that commit to a given form of production for a given target poses significant costs and challenges to vaccine manufacturers if there is a major change in strategy. Long lead times in manufacturing, and potentially hundreds of complex process steps, all complicate capabilities. Vaccine companies, like the biotech industry, desire novel production methods out of a need for efficiency that reduce the cost of goods, shorten time to licensure, and respond quicker to disease outbreaks. mRNAbased vaccines hold the promise to revolutionize the field by addressing current manufacturing challenges and offering novel vaccine compositions.

Assuming that mRNA vaccines will be proven clinically efficacious and safe, one of the central advantages hinges on rapidity of manufacture. Within weeks, clinical batches can be generated after the availability of a sequence encoding the immunogen. The process is cell-free and scalable. Of paramount advantage, a facility dedicated to mRNA production should be able to rapidly manufacture vaccines against multiple targets, with minimal adaptation to processes and formulation. In addition, new targets requiring multi-antigen approaches will benefit from the speed in which mRNA can render multiple constructs.

Beyond manufacturing advantages, mRNA technology is impacting vaccine discovery and research. Expression may be possible for complex proteins that are difficult or impossible to generate with current expression systems. ${ }^{1}$ mRNA constructs can also be used to express potent monoclonal antibodies for novel immunoprophylaxis. ${ }^{2}$ Here we review the state of the art in mRNA constructs and delivery technologies for the prevention of infectious diseases, and a review of pertinent topics to the biotechnology and pharmaceutical industries.

\section{“STATE-OF-THE-ART" mRNA CONSTRUCTS AND DELIVERY TECHNOLOGIES}

The core principle behind mRNA as a technology for vaccination is to deliver the transcript of interest, encoding one or more immunogen(s), into the host cell cytoplasm where expression generates translated protein(s) to be within the membrane, secreted or intracellularly located. Two categories of mRNA constructs are being actively evaluated: non-replicating mRNA (NRM) and self-amplifying mRNA (SAM) constructs (Fig. 1). Both have in common a cap structure, $5^{\prime}$ and $3^{\prime}$ untranslated regions (UTRs), an open-reading frame (ORF), and a $3^{\prime}$ poly(A) tail. ${ }^{3}$ SAM differs with the inclusion of genetic replication machinery derived from positive-stranded mRNA viruses, most commonly from alphaviruses such as Sindbis and Semliki-Forest viruses. ${ }^{4,5}$ Generally, the ORF encoding viral structural proteins is replaced by the selected transcript of interest, and the viral RNA-dependent RNA polymerase is retained to direct cytoplasmic amplification of the replicon construct. The potential merits of NRM versus SAM will be addressed later.

The manufacturing process begins with the generation of a plasmid DNA (pDNA) containing a DNA-dependent RNA polymerase promoter, such as $T 7,{ }^{6}$ and the corresponding sequence for the mRNA construct. The pDNA is linearized to serve as a template for the DNA-dependent RNA polymerase to transcribe the mRNA, and subsequently degraded by a DNase process step. The addition of the $5^{\prime}$ cap and the $3^{\prime}$ poly $(A)$ tail can be achieved during the in vitro transcription step $^{7,8}$ or enzymatically after transcription. ${ }^{9}$ Enzymatic addition of the cap can be accomplished by using guanylyl transferase and 2'-O-methyltransferase to yield a Cap 0 ( $\left.{ }^{\mathrm{N7Me}} \mathrm{GpppN}\right)$ or Cap 1 ( $\left.{ }^{\mathrm{N} 7 \mathrm{Me}} \mathrm{GpppN}^{2^{\prime}-\mathrm{OMe}}\right)$ structure, respectively, while the poly-A tail can be achieved through enzymatic addition via poly-A polymerase.

Purification is a crucial next step, which can be achieved with the application of high-pressure liquid chromatography (HPLC). ${ }^{10}$ The resultant drug substance is then formulated into drug product and released based on sterility, identity, purity, and potency testing. These processes allow Good Manufacturing Practise (GMPs) facilities to switch to a new vaccine within a very short

${ }^{1}$ Coalition for Epidemic Preparedness Innovations (CEPI), Gibbs building, 215 Euston Road, Bloomsbury, London NW1 2BE, UK. ${ }^{2}$ Sanofi Pasteur, 1 Discovery Dr, Swiftwater, PA 18370, USA. ${ }^{3}$ Translate Bio, 29 Hartwell Ave, Lexington, MA 02421, USA. *email: nick.jackson@cepi.net 


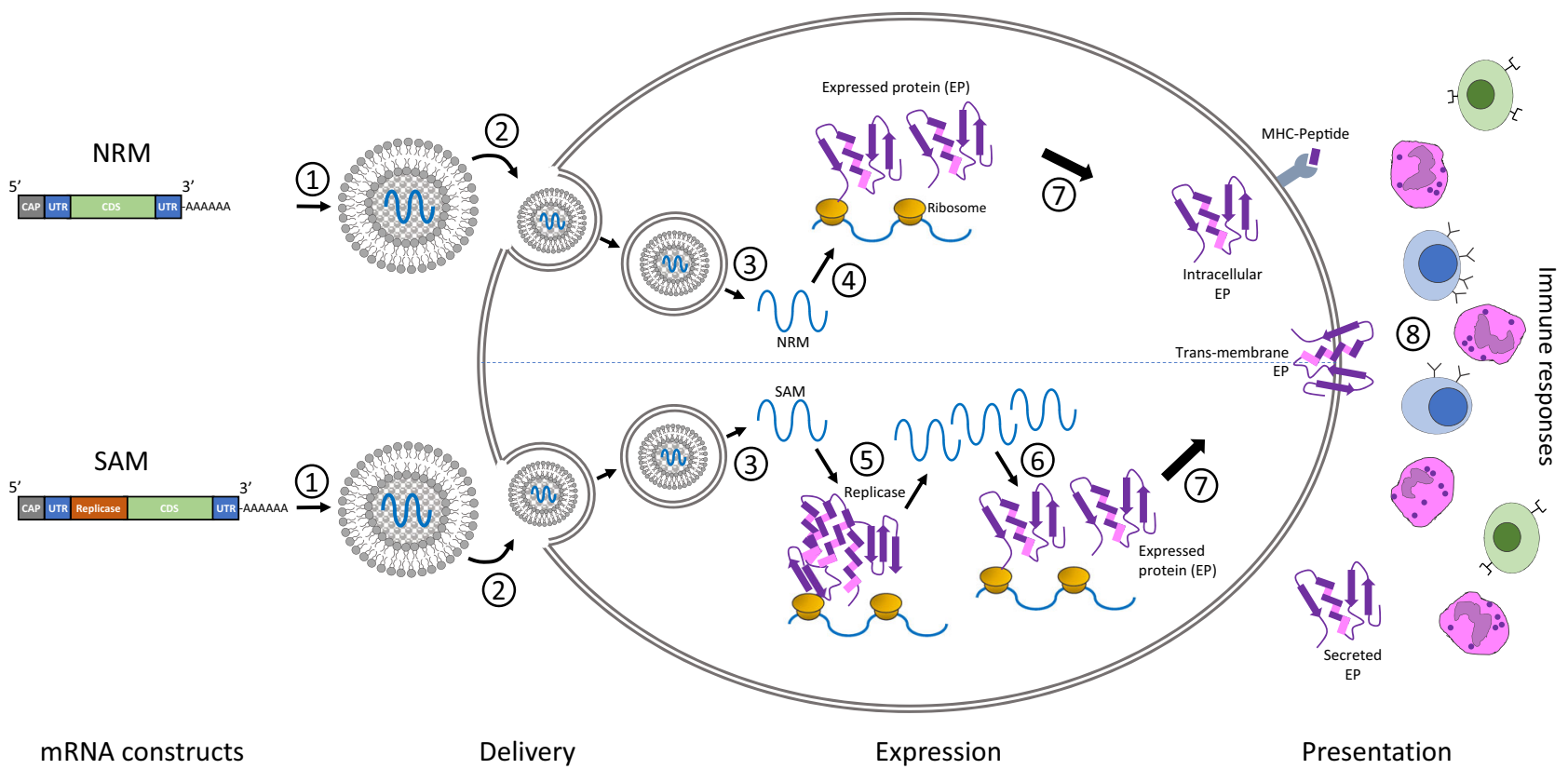

Fig. 1 Two categories of mRNA constructs are being actively evaluated. Non-replicating mRNA (NRM) constructs encode the coding sequence (CDS), and are flanked by $5^{\prime}$ and $3^{\prime}$ untranslated regions (UTRs), a $5^{\prime}$-cap structure and a $3^{\prime}$-poly-(A) tail. The self-amplifying mRNA (SAM) construct encodes additional replicase components able to direct intracellular mRNA amplification. (1) NRM and SAM are formulated in this illustration in lipid nanoparticles (LNPs) that encapsulate the mRNA constructs to protect them from degradation and promote cellular uptake. (2) Cellular uptake of the mRNA with its delivery system typically exploits membrane-derived endocytic pathways. (3) Endosomal escape allows release of the mRNA into the cytosol. (4) Cytosol-located NRM constructs are immediately translated by ribosomes to produce the protein of interest, which undergoes subsequent post-translational modification. (5) SAM constructs can also be immediately translated by ribosomes to produce the replicase machinery necessary for self-amplification of the mRNA. (6) Self-amplified mRNA constructs are translated by ribosomes to produce the protein of interest, which undergoes subsequent post-translational modification. (7) The expressed proteins of interest are generated as secreted, trans-membrane, or intracellular protein. (8) The innate and adaptive immune responses detect the protein of interest.

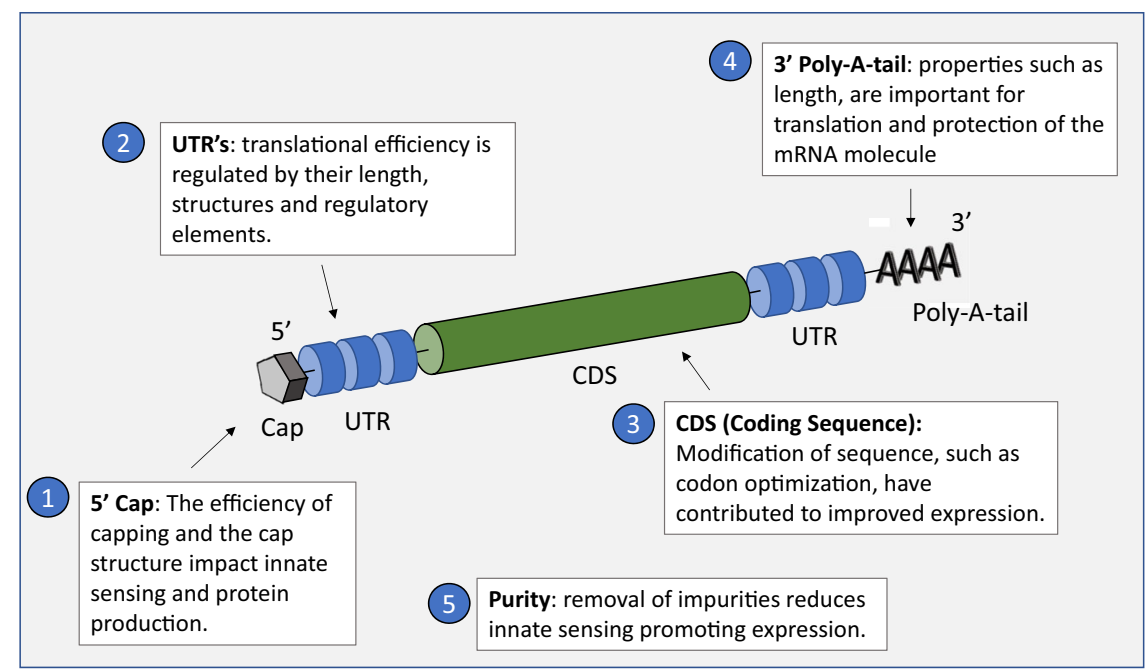

Fig. 2 Critical quality attributes (CQAs) have been identified that dictate the performance of the mRNA construct to express the gene of interest efficiently. Five principal CQAs include $5^{\prime}$ capping efficiency and structure; UTR structure, length, and regulatory elements; modification of coding sequence; poly-A-tail properties; mRNA purity.

period of time, given that the reaction materials and vessels are the same.

The design of a mRNA construct for vaccination, once released into the cytoplasm of a cell, is to efficiently utilize the translational machinery of the host cell to generate a sufficient quantity of the encoded immunogen that is presented appropriately to the immune system. Across the field, several critical quality attributes have been, and continue to be, the focus of efforts to maximize gene expression (Fig. 2). First, the purity of the mRNA is a crucial determinant of yields, and it is known that the DNA-dependent RNA polymerases yield smaller oligoribonucleotide impurities as a result of abortive initiation events, ${ }^{11}$ as well as double-stranded (ds) RNA generated by self-complementary $3^{\prime}$ extension, ${ }^{12}$ which can result in type I interferon and inflammatory cytokine production through pattern recognition receptors. Karikó et al. ${ }^{13}$ demonstrated that removal of contaminants in mRNA 
preparations reduced innate immune responses and resulted in significantly higher levels of reporter protein expression in vitro.

Second, the $5^{\prime}$ and $3^{\prime}$ UTR regions are important for maximizing gene expression. The length of the $3^{\prime}$ UTR, ${ }^{14} 5^{\prime}$ UTR structures, and regulatory elements in both UTRs ${ }^{15}$ all impact efficiency. Third, the $5^{\prime}$ 7-methylguanosine (m7G) cap of the mRNA molecule, linked via a triphosphate bridge to the first transcribed nucleotide, is essential for efficient translation, and blocks $5^{\prime}-3^{\prime}$ exonucleasemediated degradation. The specific cap structure plays a critical role in both protein production and immunogenicity, with incomplete capping ( $5^{\prime}$ triphosphate) and Cap 0 structures shown to stimulate RIG-1. ${ }^{16-18}$ In addition, 2-O'-unmethylated capped RNA can be sequestered by cellular IFN-induced proteins with tetratricopeptide repeats (IFIT1) that prevent the initiation of translation, ${ }^{19}$ or detected by the cytoplasmic RNA sensor MDA5 ${ }^{20}$ Manufacturers of mRNA vaccines pay careful attention to the choice of enzyme and reaction conditions, in order to catalyze the highest percentage of cap formation. Fourth, the poly (A) tail and its properties such as length, are crucial for translation and protection of the mRNA molecule. ${ }^{21,22}$

Last, codon optimization and modification of nucleotides have contributed to translation efficiency. For example, optimization of guanine and cytosine (GC) content can have a significant impact, ${ }^{23}$ and has been well established with DNA vaccines. The innate immune activation to mRNA can also influence its utility as a delivery system. The use of modified nucleosides, such as pseudouridine or N-1-methylpseudouridine to remove intracellular signaling triggers for protein kinase $\mathrm{R}$ (PKR) activation, resulted in enhanced antigen expression and adaptive immune responses. ${ }^{24-26}$ It has been demonstrated that successful protein production, minimal undesired inflammatory responses, and systemic adaptive immune responses could be achieved preclinically by using unmodified $\mathrm{mRNA}^{27}$ through a combination of optimizing the coding sequence and removal of any unwanted inflammatory impurities. ${ }^{28,29}$ Ultimately, comparative immunogenicity between these approaches require studies that control all potential factors, including the delivery system. Human studies "head-to-head" comparing modified and unmodified nucleoside mRNA constructs would confirm clinically relevant differences, if any. Similarly, controlled comparisons of the aforementioned NRM and SAM are needed to determine any distinctions. Until then, all the approaches-modified, unmodified, NRM, SAM, and combinations thereof-appear feasible, and are supported by preclinical data, although unmodified nucleoside constructs may be desirable for manufacturing efficiency and transcriptional fidelity. ${ }^{30,31}$

In addition to optimizing an mRNA construct, and of quintessential importance, is the delivery of the mRNA vaccine from the bolus at the injection site into the cytoplasm of cells for the initiation of translation. As mRNA is a transient molecule by nature that is susceptible to degradation primarily through nuclease activity, efficient protection is required. ${ }^{32,33}$ This has been an intense area of research in the field, for which lipid nanoparticle (LNP) formulations are currently emerging as a leading category.

LNP delivery systems serve multiple purposes in their applications. In addition to the aforementioned sustained stability imparted through protection from nuclease degradation, they also facilitate organ specificity, efficient cellular uptake, and provide endosomal escape properties that can enhance the successful delivery of the mRNA cargo to the cytoplasmic site of action. $^{34-36}$ There have been numerous examples of successful delivery of mRNA by using LNPs for therapeutic ${ }^{37-40}$ as well as vaccine applications. ${ }^{41-44}$

Much of the focus of the continued development of such LNP carrier systems involves optimization of the ionizable lipid component, with particular focus on the acid dissociation constant (pKa) and fusogenic properties (both of the ionizable component as well as helper lipid[s]), which have been demonstrated to play key roles in efficient cytoplasmic entry and release of cargo. ${ }^{45-48}$ Next-generation LNPs may include specific targeting motifs for homing and uptake by professional antigen-presenting cells, such as dendritic cells (DC). Ligands for DC receptors could be embedded on the surface of the LNPs to target these cells and promote antigen presentation to the immune system.

\section{BIOTECH AND INDUSTRIAL PERSPECTIVES}

Perspective \#1: Improved understanding of the molecular mechanisms of action will guide further improvements in mRNA constructs and formulations

Continual optimization and improvements toward developing the next generation of mRNA-based drugs are undoubtedly occurring. Sequence optimization within UTRs and coding regions of mRNA providing greater stability and/or potency can result in higher production of the desired antigen, potentially leading to a more favorable therapeutic index. ${ }^{49,50}$

Additional sites within the mRNA construct are available for optimization as well. Novel cap structures focused on base or sugar modifications have resulted in greater translational properties through increased ribosomal interaction or enhanced stability. ${ }^{51}$ Improved stability of mRNA can also be achieved through various modifications of the triphosphate bridge within the cap structure. ${ }^{52-54}$

Optimization of the carrier system can provide significant benefit as well. Substantial attention has been placed on the development of novel ionizable lipids and formulations, with improvements in cellular uptake, endosomal release, potency, and biodegradability. ${ }^{55-57}$ The combinations of all of these areas for optimization are near limitless, and success within any of these parameters can allow for beneficial effects and can provide novel approaches for vaccines to successfully prevent disease.

Perspective \#2: Translational sciences will inform preclinical and clinical studies to promote rapid downselection of constructs and formulations

A key aspect of vaccine development efforts is the goal of making early informed decisions, based on objective data that favor or disfavor a particular candidate. It is underappreciated in the field that multi-antigen vaccine approaches are a significant challenge in the decision-making process. For example, the LNP:mRNA mass ratio can be around 10:1-30:1. Thus, multi-antigen candidates necessitate a significant amount of LNP for a given dose. LNPs are known to have inherent adjuvant properties. ${ }^{58}$ Therefore, safety and tolerability may limit multi-antigen approaches, and here translational sciences are crucial for development.

There are a variety of new translational medicine tools that can be leveraged in evaluating immunity, which include in vitro human immune system models and the related organoids to improve the predictability of clinical results. ${ }^{59}$ Systems biology techniques can help in the understanding of fine differences between various NRM and SAM vaccine sequences, as well as serving as useful tools to frame sequence optimizations during iterative development schemes. ${ }^{60}$ Further, the use of functional assessments, like human challenge models, ${ }^{61}$ where the immunologic profiles of the participants and the specific details of the challenge strain(s) are well characterized-an approach mostly used to date in the evaluation of more traditional vaccinesprovides an important and powerful method to obtain early decision-making data regarding the performance of an mRNA vaccine candidate. These tools are likely quintessential for development because current data have demonstrated a poor translation between preclinical and clinical studies with an mRNA pandemic influenza vaccine. In ferrets (ID, $2 \times 50 / 100 \mathrm{mcg}$ ), nonhuman primates (ID/IM, $2 \times 400 \mathrm{mcg}$ ), and humans (IM, $2 \times$ $100 \mathrm{mcg}$ ), an H10N8-derived HA mRNA vaccine formulated with 
LNP elicited HAl titers in the range of 2000-8000, 10,000, and 70, respectively. ${ }^{62,63}$ Whether this lack of translation is a function of mRNA not having optimized quality attributes, suboptimal delivery or an inherent limitation of preclinical or in vitro translational models is not known. Understanding this is going to be key for further development.

\section{Perspective \#3: Challenges ahead for clinical trials}

While specific regulatory guidelines are lacking for the clinical development of mRNA-based vaccines, the following general principles, as outlined in overarching guidance documents, are generally sufficient to help facilitate the entry of candidate vaccines into early-phase clinical trials. At the time of writing, 12 clinical trials for mRNA-based infectious disease vaccines have been completed, or are at various stages of progression, building experience (see Table 1). All studies assess viral targets, many have been reviewed elsewhere recently. ${ }^{64}$

The current focus from a clinical perspective is to optimize the benefit (immunogenicity and efficacy) while reducing the risk (safety) profile of a candidate mRNA vaccine by optimizing the quality attributes that dictate expression and/or augmenting delivery. It is clear that immune activation can be both advantageous and potentially detrimental, and has to be titrated accordingly. Thus, early-phase clinical trials need to be designed in a way to appropriately capture the inflammatory component intrinsic to all mRNA vaccines, given that several intracellular innate immune response sensors are activated by RNA. ${ }^{65}$ Elements include measuring administration site reactions such as pain, tenderness-associated systemic reactions such as fever and malaise, and routine biochemical laboratory parameters (e.g., serum electrolytes, liver function test, and $(B C)$. These parameters, when followed closely, can be designed to develop enrollment pause rules in the event that severe tolerability issues are observed in a clinical trial. ${ }^{66}$ Detailed characterization of the immune response fully leveraging modern techniques such as transcriptomics and systems biology, in addition to traditional methods of immune monitoring, needs to be implemented. Several agency guidelines developed for the study of novel adjuvants in human subjects provide sufficient guidance that can be applied to mRNA candidate vaccines on how to monitor safety in early-phase clinical studies. ${ }^{67}$

The data from early-phase clinical studies, particularly around local and self-limiting systemic reactogenicity, have been mixed. ${ }^{68,69}$ In fact, reporting of human trials has generally concluded that new formulations are required to optimize the profile. However, these early claims need further confirmation, and in many cases, complete datasets are still awaited. As mentioned above, multi-antigen approaches will only complicate the issue of establishing acceptable tolerability.

Humoral elicited responses have been generally underwhelming, compared with the established potency in the field of protein or live attenuated vaccines. ${ }^{63,69}$ This indicates that much formulation work is still needed to achieve sufficient immunological potency of different vaccine candidates, while maintaining acceptable tolerability-but we can be encouraged by incremental progress to date. Furthermore, very limited data exist on repeat administration of mRNA vaccines in humans. These data are important as most vaccines generally require a booster dose.

As the field accrues more data from early-phase human studies, the focus of mRNA vaccines will shift from documenting local and systemic tolerability to capturing potential long-term safety. Unfortunately detecting safety signals for uncommon adverse events requires thousands of subjects. As with novel adjuvants, an adequate safety database to assure safety for candidate mRNA vaccines is likely to be in the tens of thousands range. Given that different manufacturers are pursuing different strategies to optimize their candidate vaccines, conclusions from one candidate

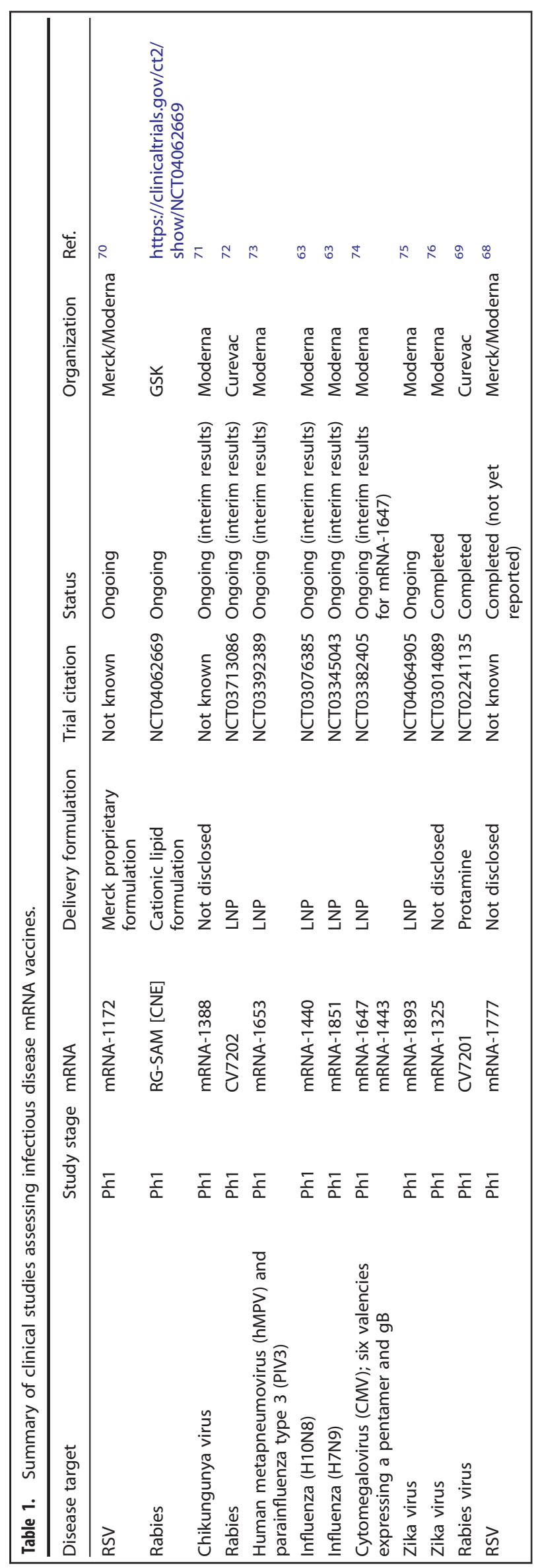


may not be generalizable. Therefore, it is likely that each candidate vaccine will have to independently prove its risk/benefit profile that is favorable.

\section{SUMMARY}

The potential advantages of mRNA as a vaccine range from the discovery of immunogens to rapid response manufacturing. Currently, the field is pursuing two approaches: non-replicating and self-replicating constructs. A number of quality attributes, that dictate stability and efficiency of expression, continue to be an intense area of development. It is widely recognized that the delivery of the mRNA into the cytoplasm is equally important to successfully elicit a robust and durable immunity. As a result, much progress has been achieved with considerable focus on novel ionizable lipid formulations and the next generation of delivery systems.

The nature of mRNA technology allows rapid refinement with almost limitless combinations of derivatives in the pursuit of optimization. This necessitates the application of translational sciences to accelerate selection of the optimal construct and formulation for subsequent development. Clinical experience in the last 2 years is building upon the plethora of preclinical data generated. These trials have informed our understanding of the need to find the optimal balance between immune and inflammatory activation to establish an acceptable risk/benefit profile for a given vaccine. Whether clinical results from different sponsors will be generalizable for mRNA technologies and formulations remains to be determined.

Overall, with significant advances in mRNA biology, delivery, and manufacturing, the biotechnology and vaccine industries are poised for further investment in the development of novel products.

Received: 9 October 2019; Accepted: 20 December 2019; Published online: 04 February 2020

\section{REFERENCES}

1. John, S. et al. Multi-antigenic human cytomegalovirus mRNA vaccines that elicit potent humoral and cell-mediated immunity. Vaccine 36, 1689-1699 (2018).

2. Kose, N. et al. A lipid-encapsulated mRNA encoding a potently neutralizing human monoclonal antibody protects against chikungunya infection. Sci. Immunol. 4, eaaw6647 (2019).

3. Pardi, N., Hogan, M. J., Porter, F. W. \& Weissman, D. mRNA vaccines-a new era in vaccinology. Nat. Rev. Drug Dis. 17, 261-279 (2018).

4. Cheng, W. F. et al. Enhancement of sindbis virus self-replicating RNA vaccine potency by linkage of herpes simplex virus type 1 VP22 protein to antigen. $J$. Virol. 75, 2368-2376 (2001).

5. Ljungberg, K. \& Liljestrom, P. Self-replicating alphavirus RNA vaccines. Exp. Rev. Vacc. 14, 177-194 (2015).

6. Rong, M., He, B., McAllister, W. T. \& Durbin, R. K. Promoter specificity determinants of T7 RNA polymerase. PNAS 95, 515-9 (1998).

7. Stepinski, J., Waddell, C., Stolarski, R., Darzynkiewicz, E. \& Rhoads, R. E. Synthesis and properties of mRNAs containing the novel "anti-reverse" cap analogs 7methyl(3'-O-methyl) GpppG and 7-methyl(3'-deoxy) GpppG. RNA 7, 1486-1495 (2001).

8. Grudzien-Nogalska, E. et al. Synthesis of anti-reverse cap analogs (ARCAs) and their applications in mRNA translation and stability. Meth. Enzym. Ch. 431, 203-227 (2007)

9. Martin, S. \& Moss, B. Modification of RNA by mRNA guanylyltransferase and mRNA (guanine 7) methyltransferase from vaccinia virions. J. Bio Chem. 250, 9330-9335 (1975).

10. Weissman, D., Pardi, N., Muramatsu, H., \& Karikó, K. HPLC Purification of in vitro transcribed long RNA. in Synthetic Messenger RNA and Cell Metabolism Modulation. Methods in Molecular Biology (Methods and Protocols) (ed. Rabinovich, P.) Vol. 969 pp 43-45 (Humana Press, Totowa, NJ, 2012).

11. Milligan, J. F., Groebe, D. R., Witherell, G. W. \& Uhlenbeck, O. C. Oligoribonucleotide synthesis using T7 RNA polymerase and synthetic DNA templates. Nucleic Acids Res. 15, 8783-8798 (1987).
12. Triana-Alonso, F. J., Dabrowski, M., Wadzack, J. \& Nierhaus, K. H. Self-coded 3'extension of run-off transcripts produces aberrant products during in vitro transcription with T7 RNA polymerase. J. Biol. Chem. 270, 6298-6307 (1995).

13. Karikó, K., Muramatsu, H., Ludwig, J. \& Weissman, D. Generating the optimal mRNA for therapy: HPLC purification eliminates immune activation and improves translation of nucleoside-modified, protein-encoding mRNA. Nucleic Acids Res. 39, e142 (2011).

14. Tanguay, R. \& Gallie, D. Translational efficiency is regulated by the length of the 39 untranslated region. Mol. Cell Bio. 16, 146-156 (1996).

15. Leppek, K., Das, R. \& Barna, M. Functional 5' UTR mRNA structures in eukaryotic translation regulation and how to find them. Nat. Rev. Mol. Cell Biol. 19, 158-174 (2018).

16. Devarkar, S. C. et al. Structural basis for m7G recognition and 2'-O-methyl discrimination in capped RNAs by the innate immune receptor RIG-I. PNAS 113, 596-601 (2016).

17. $\mathrm{Xu}, \mathrm{X}$. X. et al. RIG-I: a multifunctional protein beyond a pattern recognition receptor. Protein Cell 9, 246-253 (2018).

18. Lassig, C. \& Hopfner, K. P. Discrimination of cytosolic self and non-self RNA by RIG-I-like receptors. J. Biol. Chem. 292, 9000-9009 (2017).

19. Habjan, M. et al. Sequestration by IFIT1 impairs translation of $2^{\prime} \mathrm{O}$-unmethylated capped RNA. PLoS Pathog. 9, e1003663 (2013).

20. Züst, R. et al. Ribose $2^{\prime}$-O-methylation provides a molecular signature for the distinction of self and non-self mRNA dependent on the RNA sensor Mda5. Nat. Immunol. 12, 137-43 (2011).

21. Goldstrohm, A. C. \& Wickens, M. Multifunctional deadenylase complexes diversify mRNA control. Nat. Rev. Mol. Cell Biol. 9, 337-344 (2008).

22. Azoubel Lima, S. et al. Short poly(A) tails are a conserved feature of highly expressed genes. Nat. Struct. Mol. Biol. 24, 1057-1064 (2017).

23. Kudla, G., Lipinski, L., Caffin, F., Helwak, A. \& Zylicz, M. High guanine and cytosine content increases mRNA levels in mammalian cells. Plos Biol. 4, e180 (2016).

24. Anderson, B. et al. Incorporation of pseudouridine into mRNA enhances translation by diminishing PKR activation. Nucleic Acids Res. 38, 5884-5892 (2010).

25. Andries, O. et al. N1-methylpseudouridine-incorporated mRNA outperforms pseudouridine-incorporated mRNA by providing enhanced protein expression and reduced immunogenicity in mammalian cell lines and mice. J. Contr. Rel. 217, 337-344 (2015).

26. Pardi, N. et al. Nucleoside-modified mRNA vaccines induce potent $T$ follicular helper and germinal center B cell responses. J. Exp. Med. 215, 1571-1588 (2018).

27. Lutz, J. et al. Unmodified mRNA in LNPs constitutes a competitive technology for prophylactic vaccines. npj Vaccines 2, 29 (2017).

28. Thess, A. et al. Sequence-engineered mRNA without chemical nucleoside modifications enables an effective protein therapy with large animals. Mol. Ther. 23 1456-1464 (2015).

29. Kauffman, K. J. et al. Efficacy and immunogenicity of unmodified and pseudouridine-modified mRNA delivered systemically with lipid nanoparticles in vivo. Biomaterials 109, 78-87 (2016).

30. Potapov, V. et al. Base modifications affecting RNA polymerase and reverse transcriptase fidelity. Nucleic Acids Res. 46, 5753-5763 (2018).

31. Wu, M. et al. Effect of mRNA modifications on translational fidelity. in Proc. Poster presented at 6th International mRNA (INTERPLAN Congress, Boston, MA, 2018).

32. Tsui, N. B., Ng, E. K. \& Lo, Y. M. Stability of endogenous and added RNA in blood specimens, serum, and plasma. Clin. Chem. 48, 1647-1653 (2002).

33. Houseley, J. \& Tollervey, D. The many pathways of RNA degradation. Cell 136 763-776 (2009).

34. Kowalski, P. S., Rudra, A., Miao, L. \& Anderson, D. G. Delivery the messenger: Advances in technologies for therapeutic mRNA delivery. Mol. Ther. 27, 710-728 (2019).

35. Stanton, M. Current status of messenger RNA delivery systems. Nucleic Acids Ther. 28, 158-165 (2018).

36. Midoux, P. \& Pichon, C. Lipid-based mRNA vaccine delivery systems. Exp. Rev. Vacc. 14, 221-234 (2015).

37. DeRosa, F. et al. Improved efficacy in a Fabry disease model using a systemic mRNA liver depot system as compared to enzyme replacement therapy. Mol. Ther. 27, 878-889 (2019).

38. DeRosa, F. et al. Therapeutic efficacy in a hemophilia B model using a biosynthetic mRNA liver depot system. Gene Ther. 23, 699-707 (2016).

39. An, D. et al. Systemic messenger RNA therapy as a treatment for methylmalonic acidemia. Cell Rep. 21, 3548-3558 (2017).

40. Asrani, K. H., Cheng, L., Cheng, C. J., Subramanian, R. R. \& Arginase, I. mRNA therapy-a novel approach to rescue arginase 1 enzyme deficiency. RNA Biol. 15, 914-922 (2018).

41. Weide, B. et al. Results of the first Phase $\mathrm{I} / \mathrm{Il}$ clinical vaccination trial with direct injection of mRNA. J. Immunother. 31, 180-188 (2008).

42. Petsch, B. et al. Protective efficacy of in vitro synthesized, specific mRNA vaccines against influenza A virus infection. Nat. Biotech. 30, 1210-1218 (2012). 
43. Kallen, J. et al. A novel, disruptive vaccination technology. Self-adjuvanted RNActive vaccines. Hum. Vacc. Immunother. 9, 2263-2276 (2013).

44. Pardi, N. et al. Zika virus protection by a single low-dose nucleoside-modified mRNA vaccination. Nature 543, 248-251 (2017).

45. Sabnis, S. et al. A novel amino lipid series for mRNA delivery: improved endosomal escape and sustained pharmacology and safety in non-human primates. Mol. Ther. 26, 1509-1519 (2018).

46. Kauffman, K. J. et al. Optimization of lipid nanoparticle formulations for mRNA delivery in vivo with fractional factorial and definitive screening designs. Nano Lett. 15, 7300-7306 (2015).

47. Mok, K. W. \& Cullis, P. R. Structural and fusogenic properties of cationic liposomes in the presence of plasmid DNA. Biophys. J. 73, 2534-2545 (1997).

48. Wasungu, L. \& Hoekstra. Cationic lipids, lipoplexes and intracellular delivery of genes. J. Contr. Rel. 116, 255-264 (2006).

49. Orlandini von Niessen, A. G. et al. Improving mRNA-based therapeutic gene delivery by expression-augmenting $3^{\prime}$-UTRs identified by cellular library screening. Mol. Ther. 27, 824-836 (2019).

50. Presnyak, V. et al. Codon optimality is a major determinant of mRNA stability. Cell 160, 1111-1124 (2015).

51. Kore, A. R., Shanmugasundaram, M., Charles, I., Vlassov, A. V. \& Barta, T. J. Locked nucleic acid (LNA)-modified dinucleotide mRNA cap analogue: synthesis, enzymatic incorporation, and utilization. J. Am. Chem. Soc. 131, 6364-6365 (2009).

52. Grudzien-Nogalska, E., Jemielity, J., Kowalska, J., Darzynkiewicz, E. \& Rhoads, R. E. Phosphorothioate cap analogs stabilize mRNA and increase translational efficiency in mammalian cells. RNA 13, 1745-1755 (2007).

53. Grudzien, E. et al. Novel cap analogs for in vitro synthesis of mRNAs with high translational efficiency. RNA 10, 1479-1487 (2004).

54. Rydzik, A. M. et al. mRNA cap analogues substituted in the tetraphosphate chain with CX2: identification of $\mathrm{O}$-to- $\mathrm{CCl} 2$ as the first bridging modification that confers resistance to decapping without impairing translation. Nucleic Acids Res. 45, 8661-8675 (2017).

55. Fenton, O. S. et al. Bioinspired alkenyl amino alcohol ionizable lipid materials for highly potent in vivo mRNA delivery. Adv. Mater. 28, 2939-2943 (2016).

56. Durymanov, M. \& Reineke, J. Non-viral delivery of nucleic acids: insight into mechanisms of overcoming intracellular barriers. Front. Pharmacol. 9, 971 (2018).

57. Li, B., Zhang, X. \& Dong, Y. Nanoscale platforms for messenger RNA delivery.Wiley Interdiscip. Rev. Nanomed. Nanobiotech. 11, e1530 (2019).

58. Swaminathan, G. et al. A novel lipid nanoparticle adjuvant significantly enhances B cell and T cell responses to sub-unit vaccine antigens. Vaccine 34, 110-9 (2016).

59. Ramani, S., Crawford, S. E., Blutt, S. E. \& Estes, M. K. Human organoid cultures: transformative new tools for human virus studies. Curr. Opin. Virol. 29, 79-86 (2018).

60. Sharma, M., Krammer, F., García-Sastre, A. \& Tripathi, S. Moving from empirical to rational vaccine design in the 'Omics' Era. Vaccines 7, (E89 (2019).

61. Cooper, M. M., Loiseau, C., McCarthy, J. S. \& Doolan, D. L. Human challenge models: tools to accelerate the development of malaria vaccines. Expert Rev. Vaccines. 18, 241-251 (2019).

62. Bahl, K. et al. Preclinical and clinical demonstration of immunogenicity by mRNA vaccines against H10N8 and H7N9 influenza viruses. Mol. Ther. 25, 1326-1327 (2017).

63. Feldman, R. A. et al. mRNA vaccines against $\mathrm{H} 10 \mathrm{~N} 8$ and H7N9 influenza viruses of pandemic potential are immunogenic and well tolerated in healthy adults in phase 1 randomized clinical trials. Vaccine 37, 3326-3334 (2019).

64. Maruggi, G., Zhang, C., Li, J., Ulmer, J. B. \& Yu, D. mRNA as a transformative technology for vaccine development to control infectious diseases. Mol. Ther. 27, 757-772 (2019).

65. Tatematsu, M., Funami, K., Seya, T. \& Matsumoto, M. Extracellular RNA sensing by pattern recognition receptors. J. Innate Immun. 10, 1-9 (2018).

66. Food and Drug Administration. Guidance for Industry: Toxicity Grading Scale for Healthy Adult and Adolescent Volunteers Enrolled in Preventive Vaccine Clinical Trials. 70 FR 22664 (USA, 2007).

67. EMEA. EMEA Guidelines on Adjuvants in Vaccines for Human Use. EMEA/CHMP/ VEG/134716/2004 (EMEA, 2005).

68. Moderna reported pipeline, www.modernatx.com/pipeline.
69. Alberer, M. et al. Safety and immunogenicity of a mRNA rabies vaccine in healthy adults: an open-label, non-randomised, prospective, first-in-human phase 1 clinical trial. Lancet 390, 1511-20 (2017).

70. Moderna 2Q 2019 Report. Moderna, Inc. (2019). https://modernatx.gcs-web.com/ program-detail.

71. Shaw, C. et al. Safety and immunogenicity of a mRNA-based chikungunya vaccine in a phase 1 dose-ranging trial. Abstracts. Int. J. Infect. Dis. 79, 10.012 (2019).

72. CureVac Announces Positive Results in Low Dose $-1 \mu \mathrm{g}$ - Rabies Vaccine Clinical Phase 1 Study Curevac reported pipeline; (2020). https://www.curevac.com/ news/curevac-announces-positive-results-in-low-dose-1-\%C2\%B5g-rabiesvaccine-clinical-phase-1-study\#.

73. Moderna Announces Positive Interim Phase 1 Data for First Combination Vaccine Against the Respiratory Viruses hMPV and PIV3. (2019). https://investors. modernatx.com/news-releases/news-release-details/moderna-announcespositive-interim-phase-1-data-first.

74. Moderna Inc. R\&D Days. Exhibit 99.3 (2019) https://www.sec.gov/Archives/edgar/ data/1682852/000119312519243385/d796420dex993.htm.

75. Jagger, B. W. et al. Protective efficacy of nucleic acid vaccines against transmission of zika virus during pregnancy in mice. J. Infect. Dis. 220, 1577-1588 (2019).

76. Moderna Announces Funding Award from BARDA for 8 Million with Potentialofupto Million to Accelerate Development of Zika Messenger RNA (mRNA) Vaccine. (2016). https://investors.modernatx.com/news-releases/news-release-details/ moderna-announces-funding-award-barda-8-million-potential-125.

\section{AUTHOR CONTRIBUTIONS}

N.A.C.J., K.E.K., S.G. and F.D. wrote, reviewed, and approved this paper. D.C. reviewed and approved this paper.

\section{COMPETING INTERESTS}

N.A.C.J. was an employee and shareholder of Sanofi Pasteur at the time the paper was written, and changed employment to CEPI (a not-for-profit organization) during the peer review of the paper. K.E.K., D.C. and S.G. are employees and shareholders of Sanofi Pasteur. F.D. is an employee and shareholder of Translate Bio.

\section{ADDITIONAL INFORMATION}

Correspondence and requests for materials should be addressed to N.A.C.J.

Reprints and permission information is available at http://www.nature.com/ reprints

Publisher's note Springer Nature remains neutral with regard to jurisdictional claims in published maps and institutional affiliations.

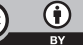

Open Access This article is licensed under a Creative Commons Attribution 4.0 International License, which permits use, sharing, adaptation, distribution and reproduction in any medium or format, as long as you give appropriate credit to the original author(s) and the source, provide a link to the Creative Commons license, and indicate if changes were made. The images or other third party material in this article are included in the article's Creative Commons license, unless indicated otherwise in a credit line to the material. If material is not included in the article's Creative Commons license and your intended use is not permitted by statutory regulation or exceeds the permitted use, you will need to obtain permission directly from the copyright holder. To view a copy of this license, visit http://creativecommons. org/licenses/by/4.0/.

(c) The Author(s) 2020 